\title{
Putting a New Spin on Scanning Transmission Electron Microscopy
}

\author{
Xiahan Sang ${ }^{1}$, Everett D. Grimley ${ }^{1}$, Changning Niu ${ }^{1}$, Douglas L. Irving ${ }^{1}$ and James M. LeBeau ${ }^{1}$ \\ 1. Department of Materials Science \& Engineering, North Carolina State University, Raleigh, NC 27606
}

Local atomic scale ordering and structural distortions can significantly modify material properties. Aberration correction dramatically improves spatial resolution into the sub-Ångstrøm regime, unlocking information about material defects previously beyond reach. While STEM has proven essential to the atomic scale characterization of materials, for example at defects, interfaces, or even in perfect crystals, measurement of atomic displacements and distances has remained challenging due to the presence of sample drift. Distortion proportional to the rate of sample drift during image acquisition hinders the accurate measurement or even representation of atomic structure. Though modern STEM installations are optimized to reduce vibration, air flow/fields, and temperature fluctuations, sample drift generally remains.

In this talk, we will introduce revolving scanning transmission electron microscopy (RevSTEM), a STEM acquisition and data processing technique designed to eliminate drift distortion [1]. The method uses a series of fast-acquisition STEM images with the scan coordinates rotated between successive frames. This scan rotation introduces a concomitant change in image distortion that we use to analyze the sample drift rate and direction. We will provide a theoretical basis for the approach and introduce the projective standard deviation (PSD) to quantify lattice vector angles in atomic resolution images. After measuring the lattice vector angles across the rotation series, the observed angular distortions are fit to a physical drift model. The resulting drift parameters are then used to calculate an inverse affine transformation matrix to restore each image.

For a demonstration of the technique, we will provide two case studies: first using images of a Si sample experiencing a significant drift rate $(530 \mathrm{pm} / \mathrm{s})$ (Figure $1 \mathrm{~b})$ and second using images of $(\mathrm{Na}, \mathrm{Bi}) \mathrm{TiO}_{3}$ with moderate drift rate but significant sample charging to evaluate the achievable accuracy and precision of the method (Figure 2). STEM images were acquired using a probe corrected FEI Titan G2 60-300 kV S/TEM equipped with an X-FEG source operated at $200 \mathrm{kV}$. The convergence and collection inner semi-angles were $21 \mathrm{mrad}$ and $77 \mathrm{mrad}$ respectively. The probe intensity was $80-100$ pA. In both cases, the RevSTEM images are shown to have minimal distortion that is comparable to the residual distortion of the scan coordinates. For Si images, more outer reflections are visible in the Fourier transform of the RevSTEM image (Figure 1b, d) than in the conventional STEM image (Figure 1a, c). The superior resolution of RevSTEM technique is demonstrated by the clear diffraction spots of $\{551\}$ planes corresponding to $0.76 \AA$ plane spacing. Moreover, the reduced dwell time for each frame in RevSTEM series can significantly reduce image tearing caused by charging, as shown in Figure 2a and $b$. RevSTEM routinely achieves 2 pm precision and sub-picometer accuracy. Finally, we will show how these features of RevSTEM unlock new possibilities to directly extract quantitative structural information when combined with detailed density functional theory calculations [2].

\section{References:}

[1] X Sang and JM LeBeau, Ultramicroscopy 138 (2014), p. 28. 
[2] The authors thank the group of Jacob Jones for providing the sample of $(\mathrm{Na}, \mathrm{Bi}) \mathrm{TiO}_{3}$. The authors acknowledge the use and support of the Analytical Instrumentation Facility at North Carolina State University, which is supported by the State of North Carolina and the National Science Foundation.
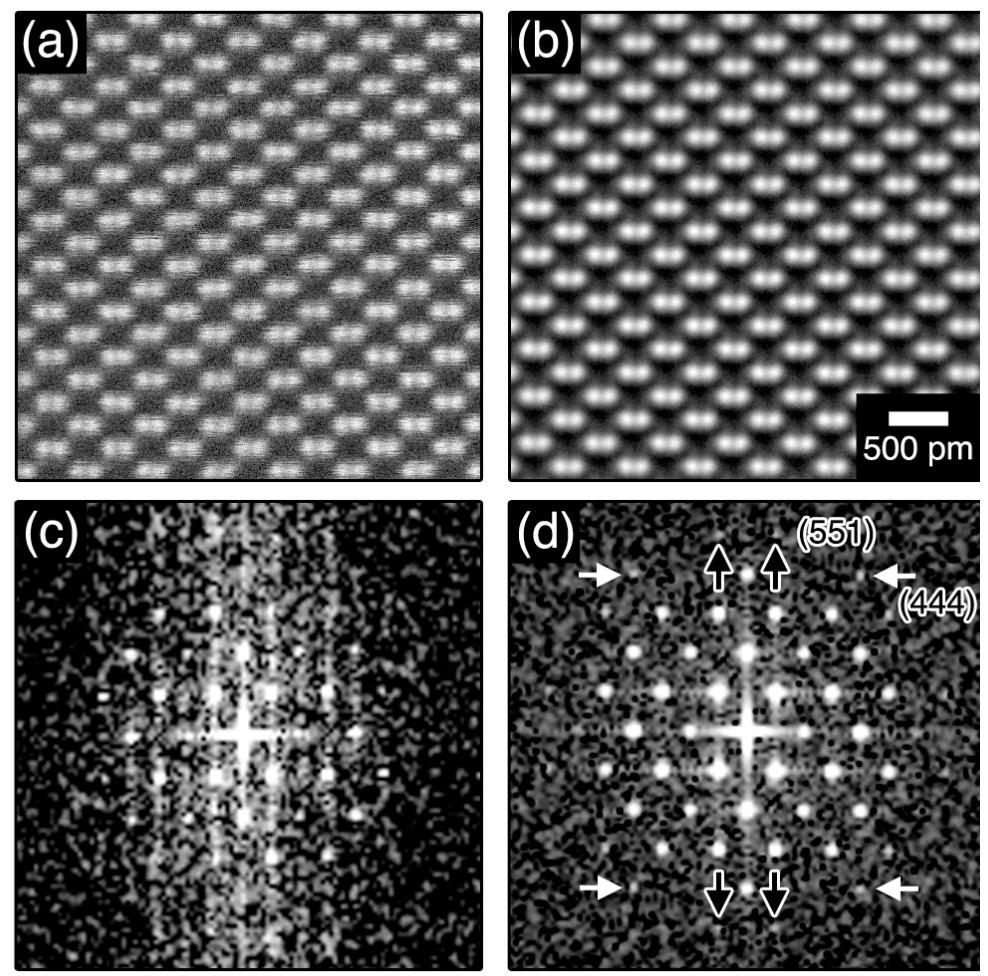

Figure 1. (a) STEM image of $\mathrm{Si}<110>$ acquired with a dwell time of $10 \mu$ s and (b) the RevSTEM average after correcting distortion of 60 frames with dwell time $3 \mu \mathrm{s}$. (c) and (d) are Fourier transform of (a) and (b) respectively using the same Hanning window. The (444) planes (white arrows, $0.783 \AA$ ) and (551) planes (black arrows, $0.76 \AA$ ) are clearly resolved in the FFT (d) of RevSTEM image (b).

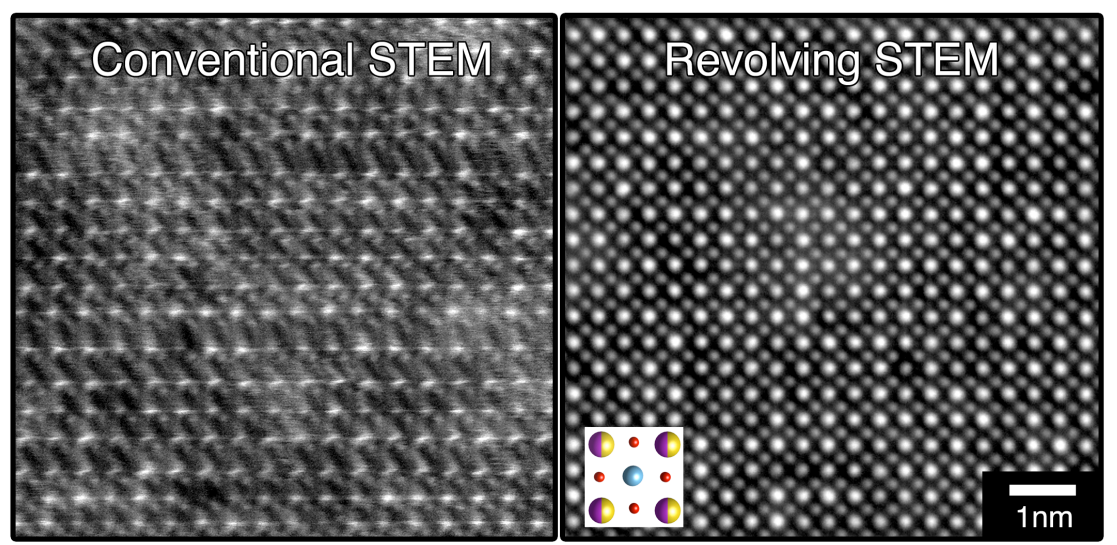

Figure 2. (a) STEM image of the $(\mathrm{Na}, \mathrm{Bi}) \mathrm{TaO} 3<100>$ acquired with a dwell time of $20 \mu \mathrm{s}$ and (b) the RevSTEM average after correcting distortion in image series of 40 frames. The dwell time for each frame was $3 \mu \mathrm{s}$. 